\title{
Tunable magnetic properties in tetragonal Mn-Fe-Ga Heusler films with perpendicular anisotropy for spintronics applications
}

\author{
Adel Kalache, ${ }^{1}$ Susanne Selle, ${ }^{2}$ Walter Schnelle, ${ }^{1}$ Gerhard H. Fecher, ${ }^{1}$ Thomas Höche, ${ }^{2}$ \\ Claudia Felser,,$^{, *}$ and Anastasios Markou ${ }^{1, \dagger}$ \\ ${ }^{1}$ Max Planck Institute for Chemical Physics of Solids, Nöthnitzer Str. 40, 01187 Dresden, Germany \\ ${ }^{2}$ Fraunhofer Institute for Microstructure of Materials and Systems IMWS, Walter-Hülse-Str. 1, 06120 Halle, Germany
}

(Received 4 May 2018; published 16 August 2018)

\begin{abstract}
High perpendicular magnetic anisotropy is essential for the development of high-efficiency spintronics devices. In this paper, we investigate the structural and magnetic properties of $\mathrm{Mn}$-Fe-Ga Heusler thin films with perpendicular magnetic anisotropy inherent to the tetragonal $D 0_{22}$ structure. High quality films were heteroepitaxially grown on $\mathrm{SrTiO}_{3}$ substrates by magnetron sputtering technique. The magnetic properties such as saturation magnetization and coercive field are easily tunable by the variation of the $\mathrm{Mn} / \mathrm{Fe}$ ratio, while retaining out-of-plane magnetization over large composition range. The uniaxial anisotropy was improved in $\mathrm{Mn}_{2.6-x} \mathrm{Fe}_{x} \mathrm{Ga}_{1.4}$ through $\mathrm{Fe}$ substitution at a fixed Ga excess. Films with composition $\mathrm{Mn}_{1.6} \mathrm{Fe}_{1} \mathrm{Ga}_{1.4}$ were found to be stable down to a thickness of $10 \mathrm{~nm}$. Transmission electron microscopy investigations proved the high quality of the films as well as chemical homogeneity. The Hall effect exhibits an anomalous contribution that dominates over the normal part, leading to Hall angle as high as $3.4 \%$. These findings suggest great potential for the integration of tetragonal Heusler materials into spintronic devices.
\end{abstract}

DOI: 10.1103/PhysRevMaterials.2.084407

\section{INTRODUCTION}

The performance of spintronic devices is largely improved with perpendicular magnetic anisotropy (PMA), i.e., where the easy axis of magnetization is aligned along the out-of-plane (OOP) axis of films [1,2]. Magnetization switching in novel nonvolatile memory, such as magnetoresistive random access memories (MRAM) [3], is expected to be ultimately driven by spin currents through the so-called spin-transfer-torque (STT) [4,5]. The advantages of such PMA devices are better thermal stability and reduced power consumption through a lower switching current. Along with PMA as a key parameter, the other material prerequisites are tunable magnetization, low Gilbert damping $\alpha$, the spin relaxation characteristics, and high Curie temperature $T_{\mathrm{C}}$ for operation well above room temperature.

Intensive efforts have been made to develop robust PMA by various approaches. The most frequently considered option, up to now, is the interfacial anisotropy arising from the close proximity of alternate layers of a ferromagnetic films, such as Fe, $\mathrm{Co}$, and $\mathrm{Ni}$, with metals like Pd or Pt having strong spin-orbit coupling [6-8]. Interfacial anisotropy relies on an accurate control of the smoothness and morphology of the layer, as well as induced strains in the magnetic layers. Alternatively, PMA

\footnotetext{
*claudia.felser@cpfs.mpg.de

† anastasios.markou@cpfs.mpg.de

Published by the American Physical Society under the terms of the Creative Commons Attribution 4.0 International license. Further distribution of this work must maintain attribution to the author(s) and the published article's title, journal citation, and DOI.
}

was also observed at the interface between magnetic metals and oxides caused by electronic hybridization [9]. Another path to PMA is achieved through the "bulk" magnetocrystalline anisotropy inherent to uniaxial crystal structures of some intermetallic compounds. Such PMA is the most reliable but requires good control of deposition parameters to manipulate structure, chemical ordering and stoichiometry.

Among spintronic-related compounds, the Heusler family constitutes a remarkable class of materials that exhibits widely tunable properties and a great functional diversity. The general full Heusler compound formula is $X_{2} Y Z$, with $X$ and $Y$ being generally transition metals, and $Z$ a main group element. Their tetragonally distorted $\mathrm{Mn}$-based relatives present tailored magnetic properties fulfilling the criteria for implementation in spintronic devices [10-15]. These Heusler compounds exhibit uniaxial magnetic anisotropy along their tetragonal $c$ axis, leading therefore to PMA in (001) oriented thin films on appropriate substrates. Ferrimagnetic $\mathrm{Mn}_{3} \mathrm{Ga}$ with $\mathrm{DO}_{22}$ structure is the most famous example which emerged as a well-suited candidate for spintronic devices, having high magnetocrystalline anisotropy, low magnetization, and small damping factor [16-19]. $\mathrm{Mn}_{3} \mathrm{Ga}$ was already considered for perpendicular magnetic tunnel junctions [20], but did not yield the high tunneling magnetoresistance ratio (TMR) expected by theory. Similarly, thin films of the $\mathrm{DO}_{22} \mathrm{Mn}_{3} \mathrm{Ge}$ phase have also attracted attention in the past years [21-25].

The structural and magnetic properties reported for bulk $\mathrm{Mn}-\mathrm{Fe}-\mathrm{Ga}$ Heusler compounds largely vary with the stoichiometry. Interesting phenomena such as exchange-spring behavior in tetragonal $\mathrm{Mn}_{2} \mathrm{FeGa}$ [26] and exchange-bias in pseudocubic $\mathrm{Fe}_{2} \mathrm{MnGa}[27,28]$ have been observed. Moreover, by tuning the $\mathrm{Mn} / \mathrm{Fe}$ ratio between these two compositions, the competing antiferromagnetic and ferromagnetic interactions 
lead to a large exchange bias observable from $2 \mathrm{~K}$ up to room temperature [29]. The $\mathrm{Mn}-\mathrm{Fe}-\mathrm{Ga}$ phase diagram was investigated through the reactive crucible melting approach for rare-earth-free permanent magnets, yielding a relatively high coercivity of $0.33 \mathrm{~T}$ for $\mathrm{Mn}_{38.5} \mathrm{Fe}_{32.5} \mathrm{Ga}_{29}$, which implies substantial magnetocrystalline anisotropy in $\mathrm{Ga}$ rich compounds [30].

The substitution of Mn by other $d$ metals in $\mathrm{Mn}_{3} \mathrm{Ga}$ films lead to ternary compounds with tailored magnetic properties. In the $\mathrm{Mn}-\mathrm{Co}-\mathrm{Ga}$ system, a transition from a tetragonal to a cubic structure occurs with increasing Co content [31] that results in a maximum anisotropy of $K_{\mathrm{U}}=1.23 \mathrm{MJm}^{-3}$ for $\mathrm{Mn}_{2.6} \mathrm{Co}_{0.3} \mathrm{Ga}_{1.1}$ [32]. A similar approach using $\mathrm{Pt}$ in $\mathrm{Mn}_{3-x} \mathrm{Pt}_{x} \mathrm{Ga}$ [33] and $\mathrm{Ru}$ in $\mathrm{Mn}_{2} \mathrm{Ru}_{x} \mathrm{Ga}$ [34,35] was adopted to obtain a zero net magnetization half-metallic Heusler material. The $\mathrm{Mn}_{2} \mathrm{RhSn}$ thin films grown on $\mathrm{MgO}$ [36] are other promising Mn-based tetragonal Heusler compounds, which display a noncollinear spin structure as ingredients for a skyrmion phase [37]. The so-called constructive chemical disorder, i.e., replacing $\mathrm{Mn}$ in $\mathrm{Mn}_{3} \mathrm{Ga}$ by $3 d$ elements has been theoretically predicted to improve the spin polarization along the tetragonal $c$ axis [38]. Thin films of $\mathrm{Mn}-\mathrm{Fe}-\mathrm{Ga}$ were recently reported to show high magnetocrystalline anisotropy; however, these films display high roughness [39]. Thin films of $\mathrm{Mn}_{2} \mathrm{Fe}_{x} \mathrm{Ga}$ grown on $\mathrm{MgO}$ substrates display a slight decrease of saturation magnetization $M_{\mathrm{s}}$ with Fe concentration $x$, however, with nonfixed Ga concentration [40]. Such films are proposed as strong candidates for STT-MRAM applications.

Our previous study on the strong PMA of Mn-Fe-Ga films grown on $\mathrm{MgO}$ shows the increase of $M_{\mathrm{s}}$ with increasing Fe substitution at fixed $\mathrm{Ga}$ concentration [41]. The growth on $\mathrm{SrTiO}_{3}$ (STO) substrates is predicted to enhance the magnetocrystalline anisotropy in $\mathrm{Mn}_{3} \mathrm{Ga}$, due to the better lattice matching as compared to $\mathrm{MgO}$ [42]. In this paper, we report on an investigation of the tunable magnetic properties of $\mathrm{Mn}-\mathrm{Fe}-\mathrm{Ga}$ thin films grown on STO substrates.

\section{EXPERIMENTAL DETAILS}

$\mathrm{Mn}-\mathrm{Fe}-\mathrm{Ga}$ thin films with a thickness of $30 \mathrm{~nm}$ were grown on single crystal STO substrates using a DC magnetron sputtering UHV system (Bestec) equipped with three targets ( $\mathrm{Mn}, \mathrm{Fe}$, and $\mathrm{Mn}_{50} \mathrm{Ga}_{50}$ ). The power for the $\mathrm{Mn}$ and Fe targets were adjusted to vary the composition of the films while the magnetron for MnGa was fixed at a certain power to keep the Ga content constant for good comparison. The resulting rate of deposition was kept around $0.1 \mathrm{~nm} / \mathrm{s}$. A fourth target was used for for the deposition of an Al capping layer with a thickness of $3 \mathrm{~nm}$ calibrated by a quartz balance. The base pressure in the chamber was always below $2 \times 10^{-8}$ mbar range. For this study, STO (001) substrates were chosen, as the lattice parameter $\left(a_{\mathrm{STO}}=3.905 \AA\right)$ fits the lattice parameter of $\mathrm{Mn}$-FeGa better than $\mathrm{MgO}\left(a_{\mathrm{MgO}}=4.212 \AA\right)$. All samples were grown at temperature of $400^{\circ} \mathrm{C}$ and at a pressure of $3 \times 10^{-3} \mathrm{mbar}$, followed by in situ annealing in vacuum for 15 minutes. Additionally, $\mathrm{Mn}_{1.6} \mathrm{Fe}_{1} \mathrm{Ga}_{1.4}$ films with different thicknesses $(20,10$, and $5 \mathrm{~nm})$ were grown in the same conditions.

Stoichiometry was determined by energy-dispersive $\mathrm{x}$-ray spectroscopy (EDXS) analysis and verified by inductively coupled plasma optical emission spectrometry (ICP-OES).

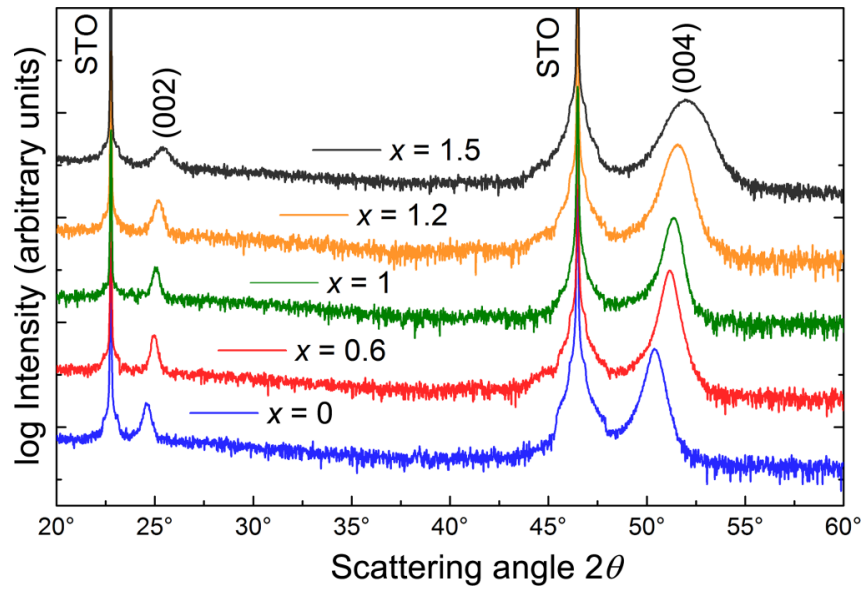

FIG. 1. XRD patterns of $\mathrm{Mn}_{2.6-x} \mathrm{Fe}_{x} \mathrm{Ga}_{1.4}$ films for different $\mathrm{Fe}$ concentration $x$.

For clarity, the chemical compositions in this study are given with the classical Heusler notation, i.e., to consistently keep four atoms per formula unit. Structural characterization was performed with a Panalytical Diffractometer XPERT ${ }^{3}$ MRD, using $\mathrm{Cu}-K_{\alpha 1}$ radiation $(\lambda=1.540 \AA$ ) to determine the lattice parameters and the full width at half maximum in rocking curve measurements (FWHM) by x-ray diffraction (XRD). The film thicknesses were studied by $x$-ray reflectivity (XRR) measurements.

Transmission electron microscopy (TEM) and scanning transmission electron microscopy (STEM) were performed using a FEI Tecnai G2 F20 microscope at $200 \mathrm{kV}$ and a FEI TITAN ${ }^{3}$ G2 80-300 microscope equipped with a SuperX EDXS analyzer at $300 \mathrm{keV}$, respectively. A protective Pt layer was deposited before the cross sectioning. TEM samples were prepared by focused ion beam (FIB) milling. The hard $\mathrm{x}$ ray photoelectron spectroscopy (HAXPES) experiments were performed at the undulator beamline BL15XU of SPring-8. The photon energy was fixed at $5.9503 \mathrm{keV}$ using a $\mathrm{Si}(111)$ double-crystal monochromator and the 333 reflection of a $\mathrm{Si}$ channel-cut post-monochromator. The photoemitted electrons were analyzed for their kinetic energy and detected by a hemispherical analyzer (VG Scienta; R4000). The overall energy resolution (monochromator plus analyzer) was set to $150 \mathrm{meV}$, as verified by spectra of the Au valence band at the Fermi energy. The detection angle was set to $2^{\circ}$ to reach the nearnormal emission geometry. Magnetization measurements were carried out using a Quantum Design magnetometer (MPMS $3)$. The magnetotransport measurements were performed on Hall bars $(1 \mathrm{~mm} \times 8 \mathrm{~mm})$ with low frequency ac current in a magnet cryostat system (PPMS, Quantum Design) with fields up to $7 \mathrm{~T}$ in the standard four-contact Hall geometry using bonded $\mathrm{Al}$ wires as contacts.

\section{RESULTS AND DISCUSSION}

\section{A. Structural characterization}

In Fig. 1, the XRD patterns of $\mathrm{Mn}_{2.6-x} \mathrm{Fe}_{x} \mathrm{Ga}_{1.4}$ thin films are shown for different Fe content. The presence of only (002) and (004) reflections demonstrates the (001) orientation of the 
TABLE I. Lattice parameters $a$ and $c$, FHWM values from the rocking curve of the (004) reflection and (112) of $\mathrm{Mn}_{2.6-x} \mathrm{Fe}_{x} \mathrm{Ga}_{1.4}$ thin films for different $\mathrm{Fe}$ concentration $x$ at $300 \mathrm{~K}$.

\begin{tabular}{lcccc}
\hline \hline & $\begin{array}{c}a \\
{[\AA]}\end{array}$ & $\begin{array}{c}c \\
{[\AA]}\end{array}$ & $\begin{array}{c}\text { FHWM (004) } \\
\mathrm{Mn}_{2.6-x} \mathrm{Fe}_{x} \mathrm{Ga}_{1.4}\end{array}$ & $\begin{array}{c}\text { FHWM (112) } \\
{\left[{ }^{\circ}\right]}\end{array}$ \\
\hline$x=0$ & 3.91 & 7.23 & 0.42 & 0.45 \\
$x=0.6$ & 3.89 & 7.13 & 0.40 & 0.32 \\
$x=1$ & 3.88 & 7.11 & 0.58 & 0.42 \\
$x=1.2$ & 3.86 & 7.08 & 0.78 & 0.52 \\
$x=1.5$ & 3.83 & 7.03 & 1.16 & 0.69 \\
\hline \hline
\end{tabular}

films with a growth along the tetragonal $c$ axis of the $\mathrm{DO}_{22}$ the Heusler cell, assuming $I 4 / \mathrm{mmm}$ space group. The theoretical site occupation for $\mathrm{Mn}_{3} \mathrm{Ga}$ would result in Ga on the $2 a(0,0$, 0 ) site, $\mathrm{Mn} 1$ on $2 b\left(0,0, \frac{1}{2}\right)$, and $\mathrm{Mn} 2$ on $4 d\left(0, \frac{1}{2}, \frac{1}{4}\right)$. From Mößbauer measurements conducted on similar compositions, it was concluded that $\mathrm{Fe}$ would preferentially occupy the $4 d$ position, with approximately $20 \%$ antisite disorder on $2 b[40,43]$. Further characterization of the ordering of the films is challenging because of the similar scattering factor of $\mathrm{Mn}$ and Fe in standard XRD, therefore limiting any precise determination of site occupancy between those two elements. The $c$ lattice parameter was directly deduced from the $d$ spacing of the (002) and (004) reflections while the $a$ lattice parameter is deduced from the position of the (112) reflection. The $c$ lattice parameter decreases from 7.23 to $7.03 \AA$, as well as the $a$ parameter from 3.91 to $3.83 \AA$, as the Fe content $x$ increases from 0 to 1.5 , as summarized in Table I. Therefore, the $c / a$ ratio remains around 1.83 for films that contain $\mathrm{Fe}$, which implies that the amount of $\mathrm{Fe}$ substitution has no significant influence on the tetragonal distortion of the $D 0_{22}$ cell. The only bulk report about the tetragonal $\mathrm{Mn}_{2} \mathrm{FeGa}$ compound [26] presents lattice parameters of $a=3.7915 \AA$ and $c=7.1858 \AA$, which corresponds to a slightly higher $c / a$ ratio (1.89 vs 1.83 in films), as compared to the off-stoichiometric $\mathrm{Mn}_{2.6-x} \mathrm{Fe}_{x} \mathrm{Ga}_{1.4}$ films. A detailed analysis of the strain in $\mathrm{Mn}-\mathrm{Fe}-\mathrm{Ga}$ films is therefore hindered, since unit cell dimensions are not available for the respective bulk reference.

Rocking curves were recorded to obtain the FWHM (full width at half-maximum) of the high intensity symmetric (004) and asymmetric (112) reflections for the tilt (grains misorientation respective to the [001] direction of substrate) and twist (grains rotation within the plane of the film) mosaicity, respectively. The FWHM of the (004) reflections is substantially influenced by the amount of Fe substitution, as depicted in Fig. 2(a). The increase of the FHWM values can be attributed to the presence of defects in the $\mathrm{DO}_{22}$ structure and/or modified growth kinetics through $\mathrm{Fe}$ substitution. The FHWM of the (112) increases as well with increasing $\mathrm{Fe}$ content, however, with slightly sharper peaks as compared to the (004) reflections of Mn-Fe-Ga films, as depicted Fig. 2(b) for the (112). Rocking curves are influenced by crystallite size and tilt mosaicity for the (004) reflection, while the (112) mainly responds to the crystallite size and the twist mosaicity. The lower value of (112) compared to (004) FWHM indicates a lower contribution from twist mosaicity compared to tilt mosaicity. Interestingly, this is not the case for the $\mathrm{Mn}_{2.6} \mathrm{Ga}$
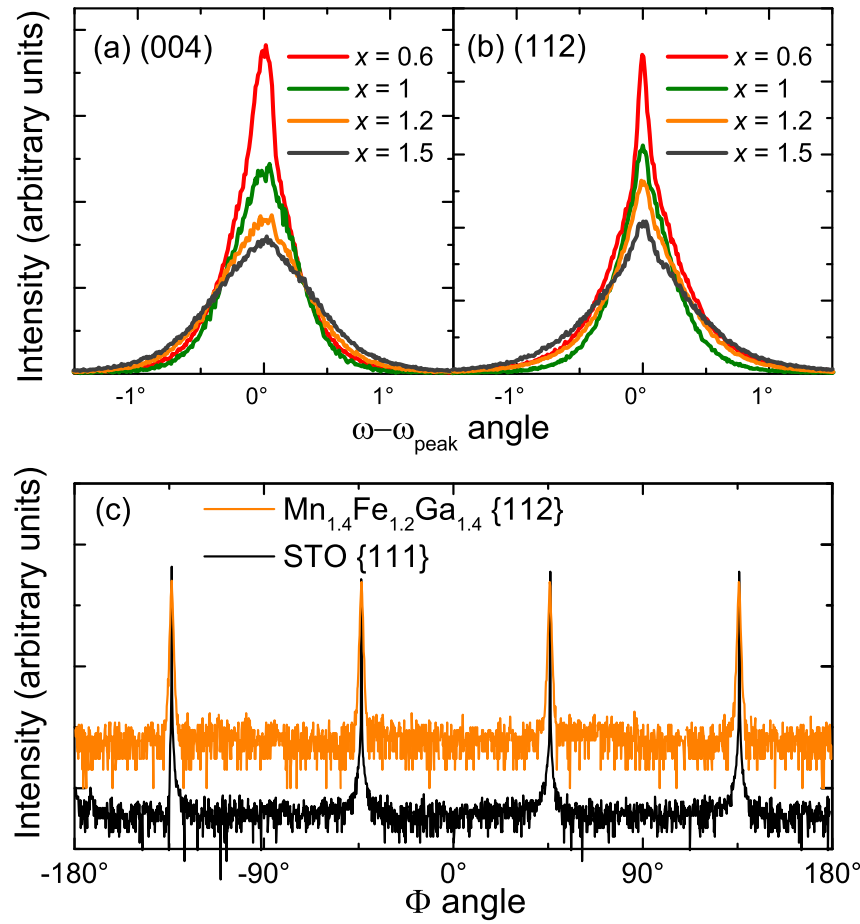

FIG. 2. Rocking curves ( $\omega$ scan) of (a) (004) and (b) (112) reflections of $\mathrm{Mn}_{2.6-x} \mathrm{Fe}_{x} \mathrm{Ga}_{1.4}$ films for different Fe content $x$ and (c) $\phi$ scans of $\{111\}$ and $\{112\}$ planes of the STO substrate and the $\mathrm{Mn}_{1.4} \mathrm{Fe}_{1.2} \mathrm{Ga}_{1.4}$ film, respectively.

film, where the tilt mosaicity appears to be scarcely prevailing. The matching of the in-plane lattice parameters of film and substrate leads to improved epitaxial growth and thus lower mosaicity as on $\mathrm{MgO}[40,41]$. For example, the film with composition $\mathrm{Mn}_{2} \mathrm{Fe}_{0.6} \mathrm{Ga}_{1.4}$ presents the lowest FWHM values of $0.40^{\circ}$ and $0.32^{\circ}$ for the symmetric (004) and asymmetric (112) reflections, respectively, as shown in Table I. This can be associated with a nearly perfect matching of the in-plane lattice parameters close to pseudomorphic growth and concomitantly reduced strains. Generally, a FWHM below $1^{\circ}$ proves good quality of heteroepitaxial film, however, the Fe substitution leads to slight deterioration of the crystalline quality. The strain relaxation state of the films might differ for the other compositions, depending on their $a$ lattice parameters, which lead to higher values of FWHM and larger mosaicity.

Furthermore, the films show fourfold symmetry as shown through $\phi$ scans for the $\mathrm{Mn}_{1.4} \mathrm{Fe}_{1.2} \mathrm{Ga}_{1.4}$ film in Fig. 2(c). The epitaxial growth is confirmed by the same occurring positions of the $\{111\}$ and $\{112\}$ set of planes of substrate and film, respectively. The heteroepitaxial relationship is therefore $\mathrm{Mn}_{1.4} \mathrm{Fe}_{1.2} \mathrm{Ga}_{1.4}[100](001) \| \mathrm{STO}[100](001)$ similar to cubeon-cube growth.

\section{B. TEM investigation}

Transmission electron microscopy investigations confirm the high quality growth of $\mathrm{Mn}-\mathrm{Fe}-\mathrm{Ga}$ thin films on STO substrates. A cross-section STEM image of the film with composition $\mathrm{Mn}_{1.6} \mathrm{Fe}_{1} \mathrm{Ga}_{1.4}$ is shown in Fig. 3(a), indicated clearly from top to down, with the protective Pt layer for FIB preparation, the $\mathrm{Al}_{x} \mathrm{O}_{y}$ capping layer, the $\mathrm{Mn}_{1.6} \mathrm{Fe}_{1} \mathrm{Ga}_{1.4}$ Heusler film, 

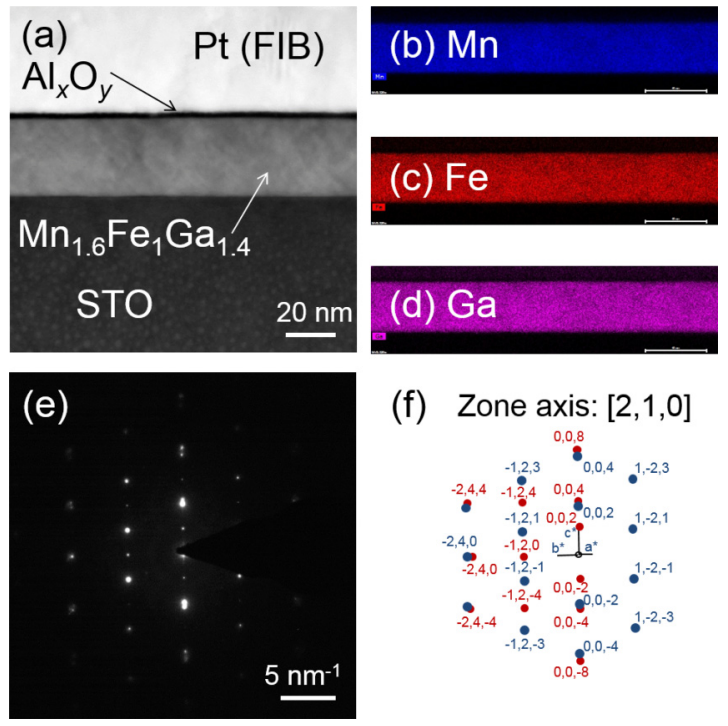

(f) Zone axis: $[2,1,0]$

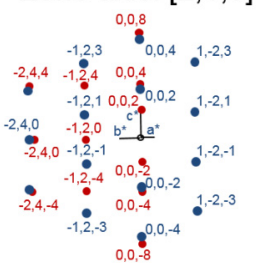

FIG. 3. (a) Cross section STEM image, and corresponding elemental mapping of (b) Mn, (c) Fe, and (d) Ga. (e) Selected area electron diffraction (SAED) pattern and (f) corresponding simulated pattern with some indexed diffraction spots of STO substrate (blue) and film (red). The arrows in (a) indicate the position of the film $\mathrm{Mn}_{1.6} \mathrm{Fe}_{1} \mathrm{Ga}_{1.4}$ and the $\mathrm{Al}_{x} \mathrm{O}_{y}$ capping layer.

and the STO substrate. The film is found to be continuous, smooth and with expected thickness of $30 \mathrm{~nm}$. The elemental distribution within the $\mathrm{Mn}_{1.6} \mathrm{Fe}_{1} \mathrm{Ga}_{1.4}$ film was analyzed by EDXS element mapping in the HAADF (high angular annular dark-field) imaging mode. The spatial distribution of the count rate intensity for $\mathrm{Mn}, \mathrm{Fe}$, and $\mathrm{Ga}$ are represented with different colors, as depicted in Figs. 3(b), 3(c), and 3(d), respectively. The three elements are homogeneously distributed over the entire film, without segregation or formation of a minority phase.

The selected area electron diffraction (SAED) pattern of the same sample is depicted in Fig. 3(e) with an electron beam parallel to the [210] zone axis. The SAED pattern is indexed with a simulated $D 0_{22}$ pattern as shown in Fig. 3(f), with identified diffraction spots of the STO substrate and the film, which are distinctly aligned. This demonstrates the single crystalline nature of the film and its heteroepitaxial growth on STO. The lattice parameters were found to be $a=$ $3.89 \AA$ and $c=7.14 \AA$, which compares well with the results from XRD. The TEM investigation proves the high quality growth of Mn-Fe-Ga films on STO substrates with excellent heteroepitaxy.

\section{HAXPES}

Hard x-ray photoelectron spectroscopy (HAXPES) is a powerful nondestructive tool for chemical, elemental, and electronic characterization of buried thin films. The spectroscopy of $2 p$ core levels allows to study the exchange interaction of the core holes with the valence electrons. The eventual oxidation of the thin films was therefore verified by HAXPES technique, which allow the investigation of buried films between the partially oxidized $\mathrm{Al}$ capping layer and the oxide substrate. HAXPES scans around Fe $2 p$ and Mn $2 p$ core

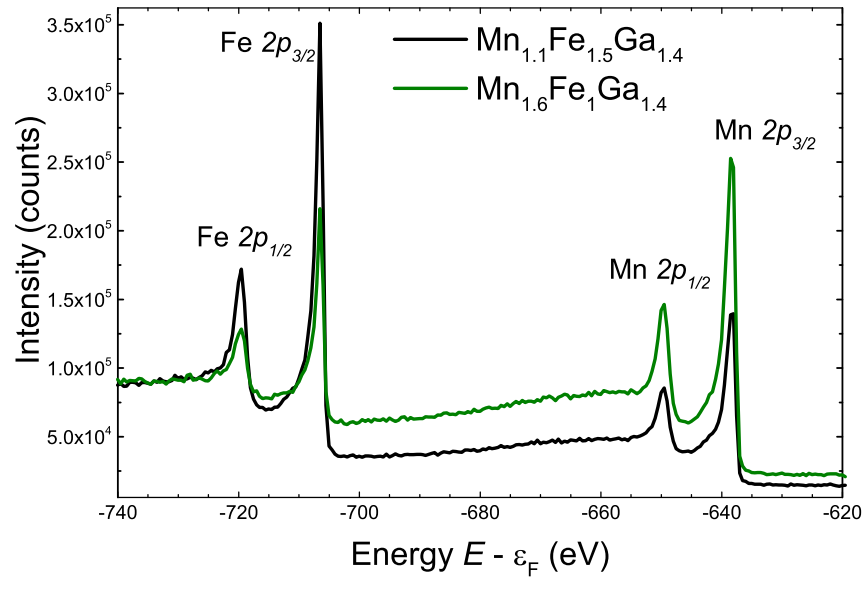

FIG. 4. Hard x-ray photoelectron spectra of thin films with $\mathrm{Mn}_{1.6} \mathrm{Fe}_{1} \mathrm{Ga}_{1.4}$ and $\mathrm{Mn}_{1.1} \mathrm{Fe}_{1.5} \mathrm{Ga}_{1.4}$ compositions.

states of two chosen different compositions $\mathrm{Mn}_{1.6} \mathrm{Fe}_{1} \mathrm{Ga}_{1.4}$ and $\mathrm{Mn}_{1.1} \mathrm{Fe}_{1.5} \mathrm{Ga}_{1.4}$ are shown in Fig. 4. The ratio of the integrated intensity of the $\mathrm{Mn} 2 p_{3 / 2}$ peak over the Fe $2 p_{3 / 2}$ peak leads to a $\mathrm{Mn} / \mathrm{Fe}$ ratio which corresponds to the composition of the investigated compositions by ICP-OES. Therefore, the HAXPES confirms the stoichiometry of the thin films for the composition $\mathrm{Mn}_{1.6} \mathrm{Fe}_{1} \mathrm{Ga}_{1.4}$ and $\mathrm{Mn}_{1.1} \mathrm{Fe}_{1.5} \mathrm{Ga}_{1.4}$. Both $2 p$ of $\mathrm{Mn}$ and $\mathrm{Fe}$ core states exhibit a spin-orbit splitting in sharp $2 p_{3 / 2}$ and $2 p_{1 / 2}$ states with $\Delta_{\mathrm{SO}(\mathrm{Mn})}=11.55 \mathrm{eV}$ and $\Delta_{\mathrm{SO}(\mathrm{Fe})}=13.15 \mathrm{eV}$ for $\mathrm{Mn}$ and $\mathrm{Fe}$, respectively, as previously observed for other Heusler compounds [44]. The absence of satellites or multiplet states confirm the metallic environment of $\mathrm{Mn}$ and $\mathrm{Fe}$ in these films, as well as Ga (not shown here). Examples for typical oxide induced satellites at the transition metal $2 p$ states are found in Ref. [45]. Therefore, the HAXPES investigation proves that the $\mathrm{Mn}-\mathrm{Fe}-\mathrm{Ga}$ thin films are not oxidized and rules out the possibility of interfacial oxidation as a source for the PMA [9].

\section{Magnetic properties}

Isothermal magnetization hysteresis loops $M(H)$ at $300 \mathrm{~K}$ for $\mathrm{Mn}-\mathrm{Fe}-\mathrm{Ga}$ thin films of different compositions are shown in Fig. 5. The OOP measurements in Fig. 5(a) display broad hard magnetic hysteresis loops, signifying an easy axis of magnetization oriented in the [001] direction of the tetragonal Heusler cell. The in-plane (IP) hysteresis loops in Fig. 5(b) indicate that the magnetic hard axis is within the plane of the films. The $\mathrm{Mn}_{2.6-x} \mathrm{Fe}_{x} \mathrm{Ga}_{1.4}$ films exhibit tunable saturation magnetization $M_{\mathrm{s}}$ from 300 to $690 \mathrm{kAm}^{-1}$ and coercivity $\mu_{0} H_{\mathrm{c}}$ from 0.76 to $0.2 \mathrm{~T}$, as the Fe content $x$ is increased from 0 to 1.5 [Figs. 6(a) and 6(b)]. Surprisingly, the coercivity is found to be larger at $x=0.6\left(\mu_{0} H_{\mathrm{c}}=0.89 \mathrm{~T}\right)$, than at $x=0$, which indicates that microstructural features are extrinsically altered by moderate Fe substitution, as observed in the FWHM values. Magnetic properties of the investigated Mn-Fe-Ga thin films are summarized in Table II.

The ferrimagnetic order of $\mathrm{Mn}_{2.6} \mathrm{Ga}_{1.4}$ is due to the antiparallel coupling of the magnetic moments of the two Mn atoms on $2 b$ and $4 d$ positions. The increase of $M_{\mathrm{s}}$ in $\mathrm{Mn}_{2.6-x} \mathrm{Fe}_{x} \mathrm{Ga}_{1.4}$ is attributed to the removal of $\mathrm{Mn}$ atoms, which reduces the 


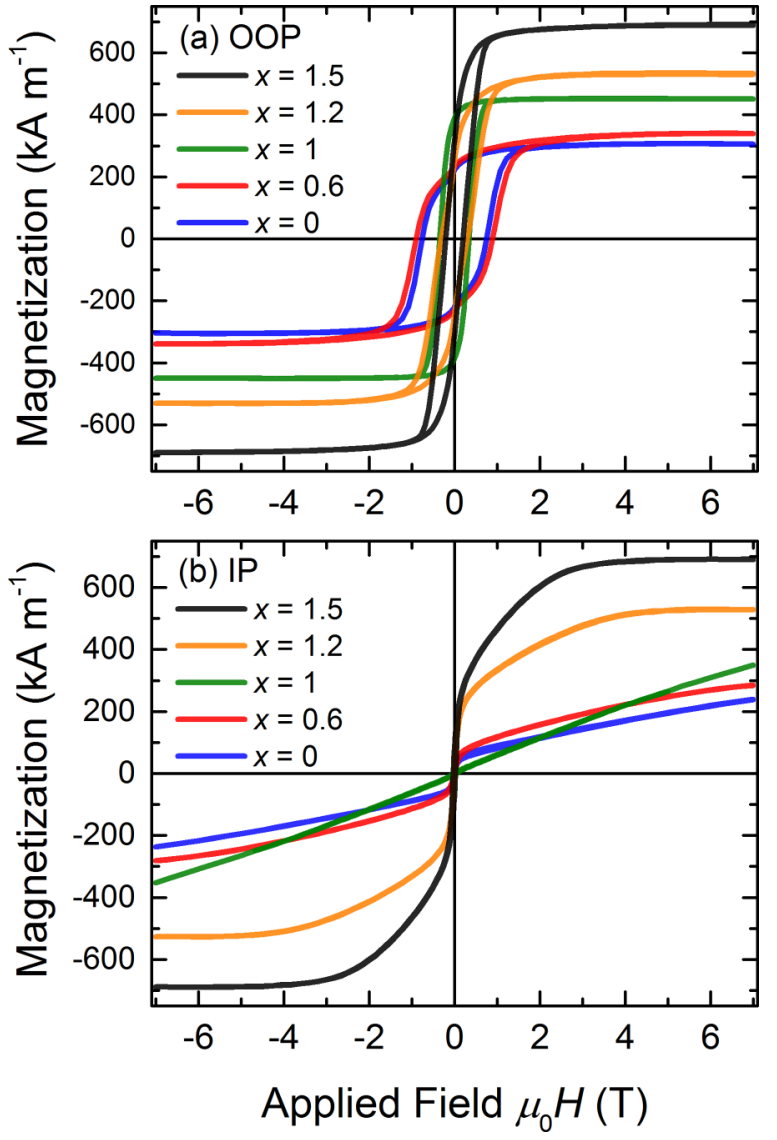

FIG. 5. Out-of-plane (a) and in-plane (b) $M(H)$ loops of $\mathrm{Mn}_{2.6-x} \mathrm{Fe}_{x} \mathrm{Ga}_{1.4}$ thin films with different $\mathrm{Fe}$ concentration $x$ at $300 \mathrm{~K}$.

antiferromagnetic interactions, whereas the $\mathrm{Fe}$ substitution leads to increased ferromagnetic coupling in the tetragonal Heusler cell, as suggested by ab initio calculations [46]. We emphasize that, for direct comparison, the Ga content has to be kept constant, as any extra Mn on the $2 b$ sites would reduce the total magnetization due to its antiferromagnetic coupling to the $\mathrm{Mn} / \mathrm{Fe}$ on $4 d$ site, as observed in $\mathrm{Mn}_{2} \mathrm{Fe}_{x} \mathrm{Ga}$ by Betto et al. [40]. The Ga excess seems to allow for a larger substitution range of $\mathrm{Fe}$ in the $\mathrm{Mn}_{2.6-x} \mathrm{Fe}_{x} \mathrm{Ga}_{1.4}$ formula, which extends the tunability of the magnetic properties. The slightly higher magnetization compared to previous thin films grown on $\mathrm{MgO}$ [41] can be attributed to the better lattice parameter matching between substrates and film and therefore

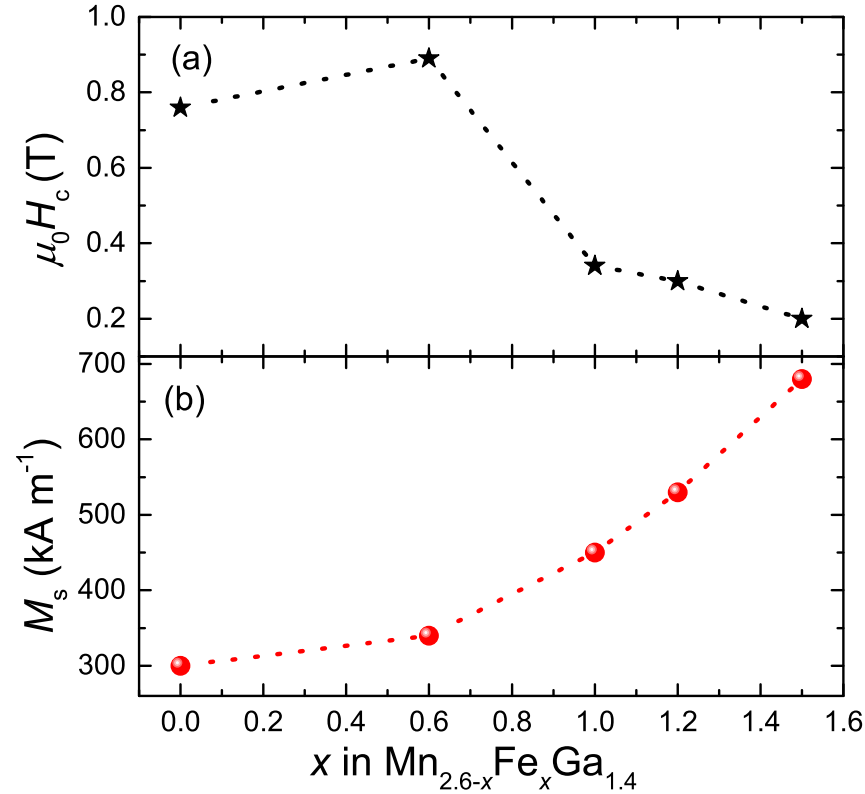

FIG. 6. Dependence of saturation magnetization $M_{\mathrm{s}}$ and coercivity $\mu_{0} H_{\mathrm{c}}$ at $300 \mathrm{~K}$ in $\mathrm{Mn}_{2.6-x} \mathrm{Fe}_{x} \mathrm{Ga}_{1.4}$ upon the Fe substitution $x$.

improved crystallinity, as shown for MnGa on LSAT substrates [47]. It also should be noted that the squareness ratio $M_{\mathrm{r}} / M_{\mathrm{s}}$ has a maximum of 0.86 for the $\mathrm{Mn}_{1.6} \mathrm{Fe}_{1} \mathrm{Ga}_{1.4}$ composition. Squareness ratio is reduced to around 0.5 for higher Fe content $x$ of 1.2 and 1.5, which implies the magnetic anisotropy is insufficient with respect to the large magnetization to develop higher remanence in Fe-rich samples. The remanence strongly depends on the microstructural features (grain size, defects) evidenced by the rocking curves, and might be improved by the use of a buffer layer. Most interestingly, our results demonstrate that the magnetic properties in tetragonal $\mathrm{Mn}-\mathrm{Fe}-\mathrm{Ga}$ films are tunable over a wide range depending on the $\mathrm{Fe}$ concentration at a fixed $\mathrm{Ga}$ excess.

The uniaxial anisotropy in thin films is estimated from $K_{\mathrm{u}}=$ $\mu_{0} M_{\mathrm{s}} H_{\mathrm{K}} / 2$, where $H_{\mathrm{K}}$ is the effective anisotropy field. Since the applied field of $7 \mathrm{~T}$ was not sufficient to saturate in-plane $M(H)$ loops of Fe-depleted Mn-Fe-Ga films, the anisotropy field $H_{\mathrm{K}}$ is estimated from linear extrapolation to the $M_{\mathrm{s}}$ value in the perpendicular direction. As the magnetization increases with the Fe concentration $x$, the anisotropy field decreases, from an estimated value around $9 \mathrm{~T}$ to measured values of

TABLE II. Saturation magnetization $M_{\mathrm{s}}$, remanence $M_{\mathrm{r}}$, coercive field $\mu_{0} H_{\mathrm{c}}$, uniaxial anisotropy $K_{\mathrm{u}}, M_{\mathrm{r}} / M_{\mathrm{s}}$ ratio as well as the remanence obtained from Hall measurement $\rho_{x y}^{r} / \rho_{x y}^{\text {sat }}$, residual resistivity ratio RRR $\left(\rho_{x x}^{300 \mathrm{~K}} / \rho_{x x}^{10 \mathrm{~K}}\right)$ and Hall angle $\left(\rho_{x y}^{\mathrm{sat}} / \rho_{x x}^{300 \mathrm{~K}}\right) \mathrm{of}^{\mathrm{Mn}} \mathrm{M}_{2.6-x} \mathrm{Fe}_{x} \mathrm{Ga}_{1.4}$ thin films for different Fe concentration $x$ at $300 \mathrm{~K}$.

\begin{tabular}{lccccccc}
\hline \hline & $\begin{array}{c}M_{\mathrm{s}} \\
{\left[\mathrm{kAm}^{-1}\right]}\end{array}$ & $\begin{array}{c}M_{\mathrm{r}} \\
{\left[\mathrm{kAm}^{-1}\right]}\end{array}$ & $\begin{array}{c}\mu_{0} H_{\mathrm{c}} \\
{[\mathrm{T}]}\end{array}$ & $\begin{array}{c}K_{\mathrm{u}} \\
{\left[\mathrm{MJm}^{-3}\right]}\end{array}$ & $\begin{array}{c}M_{\mathrm{r}} / M_{\mathrm{s}} \\
\mathrm{Mn}_{2.6-x} \mathrm{Fe}_{x} \mathrm{Ga}_{1.4}\end{array}$ & $\begin{array}{c}\rho_{x y}^{r} / \rho_{x y}^{\text {sat }} \\
\text { RRR }\end{array}$ & $\begin{array}{c}\text { Hall angle } \\
{[\%]}\end{array}$ \\
\hline$x=0$ & 300 & 220 & 0.76 & 1.42 & 0.73 & 0.90 & 1.79 \\
$x=0.6$ & 340 & 231 & 0.89 & 1.48 & 0.68 & 0.83 & 1.22 \\
$x=1$ & 450 & 385 & 0.34 & 1.75 & 0.86 & 0.77 & 1.15 \\
$x=1.2$ & 530 & 265 & 0.3 & 1.4 & 0.5 & 0.69 & 1.09 \\
$x=1.5$ & 680 & 330 & 0.2 & 1.14 & 0.49 & 0.54 & 1.07 \\
\hline \hline
\end{tabular}




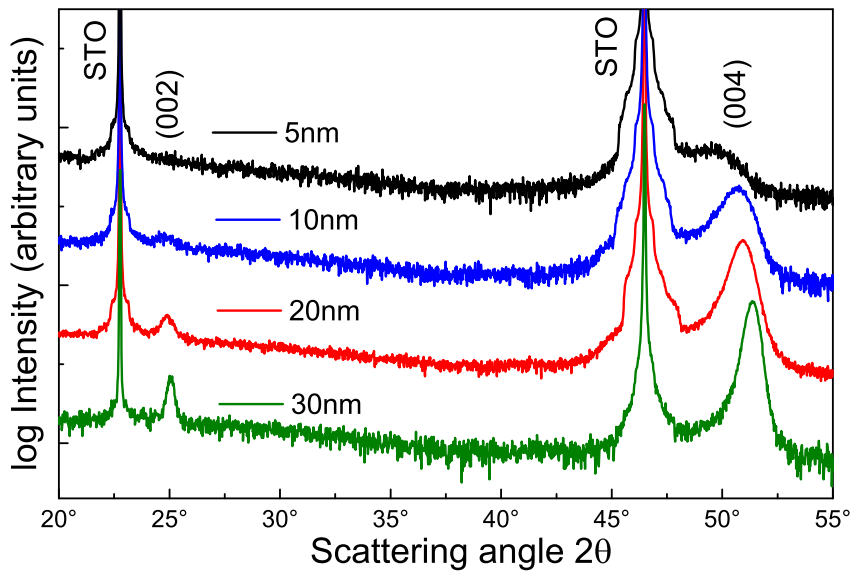

FIG. 7. XRD patterns of $\mathrm{Mn}_{1.6} \mathrm{Fe}_{1} \mathrm{Ga}_{1.4}$ films for thicknesses of $30,20,10$, and $5 \mathrm{~nm}$.

3 T. $K_{\mathrm{u}}$ in $\mathrm{Mn}_{2.6-x} \mathrm{Fe}_{x} \mathrm{Ga}_{1.4}$ varies as function of $x$ and its maximum of $1.75 \mathrm{MJm}^{-3}$ occurs at $x=1$, as a result of the interplay between $M_{\mathrm{S}}$ and $H_{\mathrm{K}}$. While the anisotropy field is reduced, the increase of magnetization allows for a substantial $K_{\mathrm{u}}$ in the Fe-rich thin films, as summarized in Table II. However, all compositions show robust values over $1 \mathrm{MJm}^{-3}$, with the main characteristic of highly tunable $M_{\mathrm{s}}, \mu_{0} H_{\mathrm{c}}$, and $H_{\mathrm{K}}$.

Scaling down the dimension in tetragonal Heusler films remains one major challenge for technological applications, due to the deterioration of the anisotropy with reduced thickness [48]. Therefore, the thickness dependence of the composition $\mathrm{Mn}_{1.6} \mathrm{Fe}_{1} \mathrm{Ga}_{1.4}$ was investigated. The XRD patterns of films with different thicknesses are shown in Fig. 7. Reducing the thickness of the films leads to pronounced peaks broadening and vanishing of the (002) reflections, as well as an increase of the $c$ lattice parameter from $7.11 \AA$ to around $7.34 \AA$. This is due to the decrease of the crystallite size as well as increase influence of strains while reducing the thickness.

The OOP and IP hysteresis loop are shown in in Fig. 8(a) for the films with reduced thicknesses. The magnetization has been normalized due to the increased error in the determination of $M_{\mathrm{S}}$ for small thicknesses. Robust PMA is clearly observed in the OOP hysteresis loop down to the 10-nm film, which retains its coercive field, as depicted in Fig. 8(b). The reduction of the thickness causes a gradual deterioration of the remanence, as shown in Fig. 8(c). The squareness ratio $M_{\mathrm{r}} / M_{\mathrm{S}}$ is reduced from 0.86 to 0.54 as the thickness is reduced from $30 \mathrm{~nm}$ to $10 \mathrm{~nm}$ thicknesses. The tetragonal distortion of the Heusler cell is altered by the increase in $c$ parameter as well as different extent of strain relaxation, which leads to gradual deterioration of the PMA. Accordingly, the effective anisotropy field $H_{\mathrm{K}}$ of the IP decreases wile reducing the thickness. However, the high uniaxial anisotropy is retained in thiner films, with an estimated value of $K_{\mathrm{u}}=0.9 \mathrm{MJm}^{-3}$ for the 10-nm film.

The rapid decrease of $\mu_{0} H_{\mathrm{c}}$ and $M_{\mathrm{r}} / M_{\mathrm{s}}$ of the 5-nm film indicates that the loss of PMA occurs around this thickness regime. The IP hysteresis loop reveals an easy axis of the magnetization of the 5-nm film, shown in Fig. 8(a). However, the OOP $M(H)$ loop of the 5-nm film presents a residual coercive field of about $50 \mathrm{mT}$, which might originate from competing
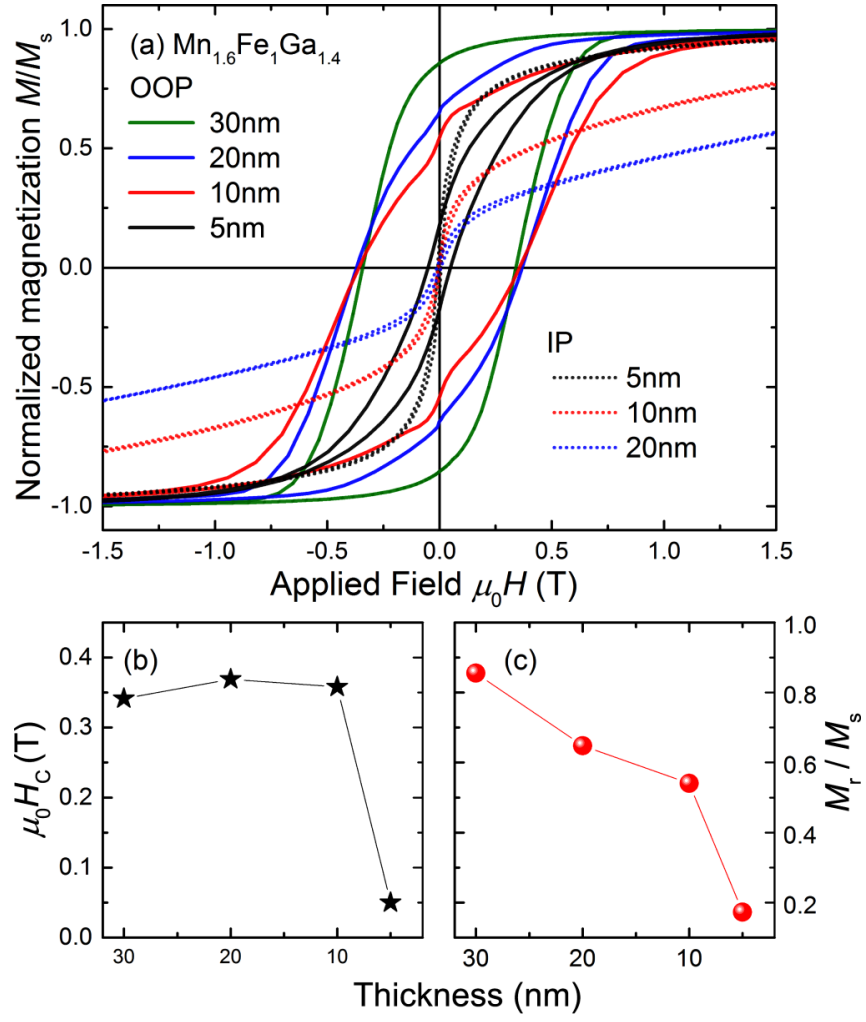

FIG. 8. Out-of-plane (solid line) and in-plane (dots) $M(H)$ curve of $\mathrm{Mn}_{1.6} \mathrm{Fe}_{1} \mathrm{Ga}_{1.4}$ thin films for thicknesses of 30, 20, 10, and $5 \mathrm{~nm}$ and thickness dependance of (b) coercivity $\mu_{0} H_{\mathrm{c}}$ and (c) $M_{\mathrm{r}} / M_{\mathrm{s}}$ ratio at $300 \mathrm{~K}$.

shape and magnetocrystalline anisotropy. The degradation of the PMA might be attributed to different combined effects, such as the increase of the $c$ parameter, the increased influence of strains, or microstructural features such as size and morphology of the grains as well as possible defects. Downscaling to less than $10 \mathrm{~nm}$ would therefore require further optimization such as a buffer layer like Pt [48] or CoGa [49] as demonstrated for $\mathrm{Mn}-\mathrm{Ga}$ to improve the squareness ratio and to retain PMA in ultrathin film.

\section{E. Magnetotransport properties}

The temperature dependence between 10 and $300 \mathrm{~K}$ of the resistivity $\rho_{x x}$ is depicted in Fig. 9 for different $\mathrm{Fe}$ concentrations $x$ in $\mathrm{Mn}_{2.6-x} \mathrm{Fe}_{x} \mathrm{Ga}_{1.4}$. The $\rho_{x x}$ increases with temperature, which indicates a metallic behavior of the films. The consistent increase of $\rho_{x x}$ with $x$ can be attributed to the chemical disorder induced by the introduction of additional scattering centers. The residual resistivity ratio (RRR) was calculated by the ratio of $\rho_{x x}^{300 \mathrm{~K}} / \rho_{x x}^{10 \mathrm{~K}}$, where the values are summarized in Table II. RRR decreases with $x$, which points to disorder induced by the introduction of $\mathrm{Fe}$ in the $\mathrm{DO}_{22}$ structure. This behavior strongly suggests a reduced crystallinity through $\mathrm{Fe}$ substitution relative to $\mathrm{Mn}_{2.6} \mathrm{Ga}_{1.4}$.

For further characterization of the PMA, magnetotransport measurements were performed in the Hall geometry. The Hall effect of a ferromagnet is usually described as the sum of two terms, $\rho_{x y}=R_{0} H+R_{\mathrm{S}} M$, where $R_{0}$ is the ordinary Hall 


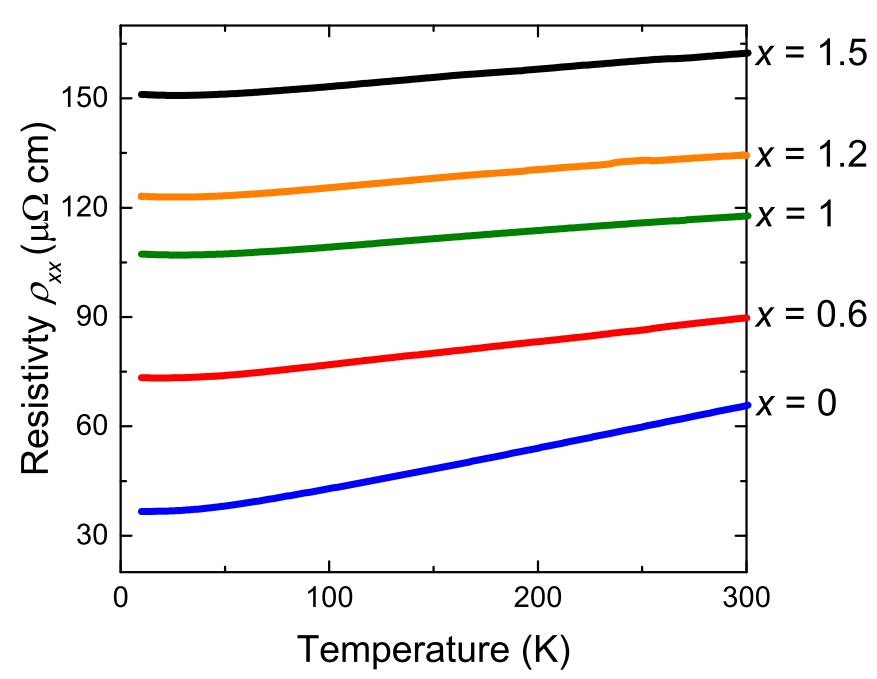

FIG. 9. Resistivity $\rho_{x x}(T)$ of $\mathrm{Mn}_{2.6-x} \mathrm{Fe}_{x} \mathrm{Ga}_{1.4}$ films for different Fe concentration $x$.

coefficient and $R_{\mathrm{S}}$ the anomalous Hall coefficient. The field dependence of the Hall resistivity $\rho_{x y}(H)$ demonstrates that the main contribution is from the anomalous Hall effect (AHE) with a minor influence from the ordinary Hall resistance $R_{0}$ in all compositions (see Fig. 10). The $\rho_{x y}(H)$ loops scale roughly with the $M(H)$ loops, with an increased Hall resistivity and a decreased coercivity as the Fe content is increased.

Moreover, the minor kinks seen in the Fe-depleted compositions $M(H)$ loops are not observed in the AHE loops, which generally exhibit improved remanence as compared to the $M(H)$ loops. The remanence over saturation in the $\rho_{x y}(H)$ loop are summarized in Table II. $\rho_{x y}^{r} / \rho_{x y}^{\text {sat }}$ is found to be larger than the $M_{\mathrm{r}} / M_{\mathrm{S}}$ ratio obtained in the $M(H)$ loops. A possible explanation given by Betto et al. [40] is, that the Mn on the $2 b$ position with a lower anisotropy does not significantly contribute to the Fermi level density of states. The AHE loops

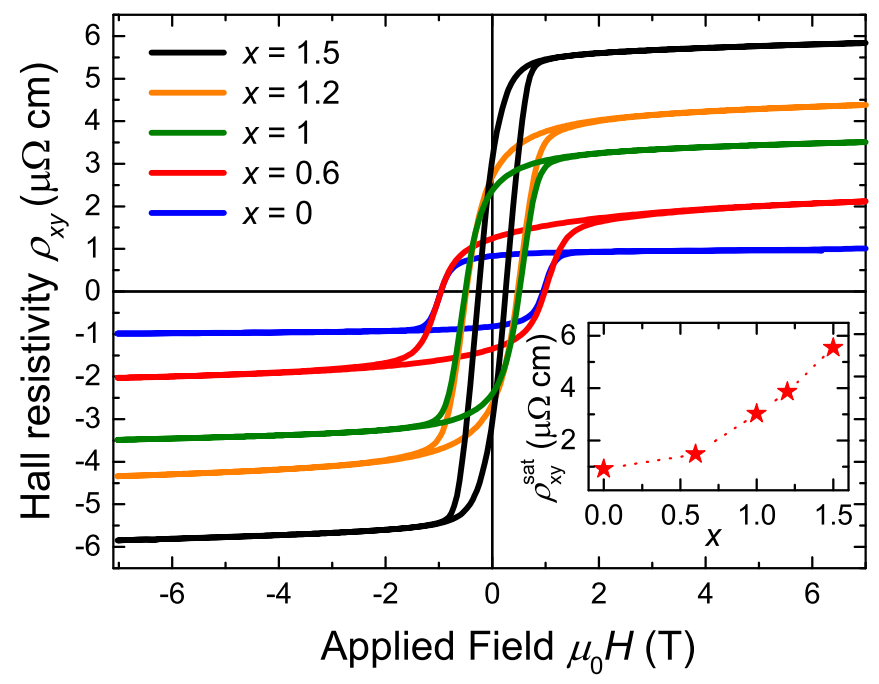

FIG. 10. Hall resistivity $\rho_{x y}$ loops of $\mathrm{Mn}_{2.6-x} \mathrm{Fe}_{x} \mathrm{Ga}_{1.4}$ films for different Fe content $x$ at $300 \mathrm{~K}$. The inset shows the dependence of $\rho_{x y}^{\text {sat }}$ upon the Mn substitution by $\mathrm{Fe}(x)$. are therefore substantially influenced by the $\mathrm{Mn} / \mathrm{Fe}$ species on the $4 d$ site, which is recognized as the site contributing the most to the magnetocrystalline anisotropy in $\mathrm{DO}_{22} \mathrm{Mn}_{3} \mathrm{Ga}$ [42,50,51].

The inset to Fig. 10 shows the dependence of saturated anomalous Hall resistivity $\rho_{x y}^{\text {sat }}$, corrected for the ordinary Hall effect, as a function of $x$. The Hall angle was estimated by the ratio of the Hall resistivity and the resistivity $\rho_{x y}^{\text {sat }} / \rho_{x x}^{300 \mathrm{~K}}$ (cf. Fig. 9). The Hall angle exhibits large values and increases with the concentration of $\mathrm{Fe} x$, from $1.40 \%$ at $x=0$ to $3.42 \%$ at $x=1.5$ (cf. Table II). These values compare in magnitude to previous reports for similar compositions, for example $2.5 \%$ in $\mathrm{Mn}_{2} \mathrm{FeGa}$ [40]. However, in this report, the Hall angle does not vary as much with $x$ in the $\mathrm{Mn}_{2} \mathrm{Fe}_{x}$ Ga formula [40]. On the contrary, the $\mathrm{Mn}_{2.6-x} \mathrm{Fe}_{x} \mathrm{Ga}_{1.4}$ films show a large increase of the Hall angle with $x$. We attribute this major difference in the Hall angle dependence to the Ga content variation. In our study, a fixed excess of $\mathrm{Ga}$ leads to enhanced magnetization in $\mathrm{Mn}_{2.6-x} \mathrm{Fe}_{x} \mathrm{Ga}_{1.4}$ thin films and therefore higher Hall angle values for Fe-rich films.

\section{SUMMARY AND CONCLUSION}

Thin films of the tetragonal Heusler compounds $\mathrm{Mn}_{2.6-x} \mathrm{Fe}_{x} \mathrm{Ga}_{1.4}$ with $\mathrm{Fe}$ concentration $x$ varying between 0 and 1.5 were grown epitaxially on STO substrates. The structural characterization confirmed the $\mathrm{DO}_{22}$ structure and the preferred (001) growth on STO substrates. TEM investigation proved that these films are single crystalline with epitaxial growth on STO, as well as the chemical homogeneity by EDXS analysis. HAXPES measurements exclude any oxidation of the film and demonstrate the metallic environment of $\mathrm{Mn}$ and $\mathrm{Fe}$ species.

The resulting magnetic properties of $\mathrm{Mn}_{2.6-x} \mathrm{Fe}_{x} \mathrm{Ga}_{1.4}(0 \leqslant$ $x \leqslant 1.5$ ) show widely tunable values of $M_{\mathrm{s}}$ and $\mu_{0} H_{\mathrm{c}}$ with robust PMA over $1 \mathrm{MJm}^{-3}$ for all $x$. Excess of Ga allows to retain the PMA and tune the magnetic properties over a wide range of $\mathrm{Fe}$ substitution. The optimized composition $\mathrm{Mn}_{1.6} \mathrm{Fe}_{1} \mathrm{Ga}_{1.4}$ shows the maximum magnetic anisotropy and the PMA could be retained down to a thickness of $10 \mathrm{~nm}$. The Hall effect was found to be dominated by the anomalous contribution. The composition dependence of AHE scales roughly with the magnetization, resulting in maximum Hall angle of $3.42 \%$ for the $\mathrm{Mn}_{1.1} \mathrm{Fe}_{1.5} \mathrm{Ga}_{1.4}$ film.

These results enrich the understanding of PMA in tetragonally distorted Heusler compounds and emphasize the importance of the main group element concentration to retain the anisotropy, in this case a slight excess of Ga. The sustained high uniaxial anisotropy upon Fe substitution demonstrates the potential of $\mathrm{Mn}-\mathrm{Fe}-\mathrm{Ga}$ thin films for integration into spintronic devices. The increased magnetization might help to resolve issues caused by the termination layer compensation in TMR devices with ferrimagnetic Mn-based Heusler [21], for example through engineering a Fe-rich $\mathrm{Mn}-\mathrm{Fe}-\mathrm{Ga}$ interface that results in ferromagnetic coupling at the proximity of the tunneling barrier.

The highly tunable magnetic properties open new possibilities to build a full Heusler TMR stack with inherent advantages of fitting lattice parameters and matching bands at the Fermi level, due to the flexibility within the tetragonal 
Heusler structure. For example, a reference layer, constituting of a hard magnetic Fe-depleted Mn-Fe-Ga Heusler, could be combined with a free layer using a Fe-rich composition, where both layers show PMA. Lastly, a Heusler semiconductor spacer layer would be preferred over insulating MgO. Such GMR or TMR stacks composed entirely of Heusler compounds are promising heterostructures to build high-efficiency spintronic devices.

\section{ACKNOWLEDGMENTS}

This work has been funded by the Joint Initiative for Research and Innovation within the Fraunhofer and Max Planck cooperation program. The synchrotron-based HAXPES measurements were performed at BL15XU of SPring-8 with the approval of the NIMS synchrotron X-ray station (Proposal No. 2015A 4910). The authors thank S. Ueda (BL15XU) for help with the experiments and S. Ouardi for fruitful discussions.
[1] S. Mangin, D. Ravelosona, J. A. Katine, M. Carey, B. Terris, and E. E. Fullerton, Nat. Mater. 5, 210 (2006).

[2] B. Tudu and A. Tiwari, Vacuum 146, 329 (2017).

[3] S. S. P. Parkin, K. P. Roche, M. G. Samant, P. M. Rice, R. B. Beyers, R. E. Scheuerlein, E. J. O’Sullivan, S. L. Brown, J. Bucchigano, D. W. Abraham, Y. Lu, M. Rooks, P. L. Trouilloud, R. A. Wanner, and W. J. Gallagher, J. Appl. Phys. 85, 5828 (1999).

[4] L. Berger, Phys. Rev. B 54, 9353 (1996).

[5] J. Slonczewski, J. Magn. Magn. Mater. 159, L1 (1996).

[6] P. F. Carcia, A. D. Meinhaldt, and A. Suna, Appl. Phys. Lett. 47, 178 (1985).

[7] M. J. Johnson, P. J. H. Bloemen, F. J. A. den Broeder, and J. J. de Vries, Rep. Prog. Phys. 59, 1409 (1996).

[8] A. Markou, I. Panagiotopoulos, T. Bakas, P. Postolache, L. Stoleriu, and A. Stancu, J. Appl. Phys. 112, 123914 (2012).

[9] B. Dieny and M. Chshiev, Rev. Mod. Phys. 89, 025008 (2017).

[10] J. Winterlik, S. Chadov, A. Gupta, V. Alijani, T. Gasi, K. Filsinger, B. Balke, G. Fecher, C. Jenkins, F. Casper, J. Kübler, G. Liu, L. Gao, S. S. P. Parkin, and C. Felser, Adv. Mater. 24, 6283 (2012).

[11] T. Graf, C. Felser, and S. S. Parkin, Prog. Solid State Chem. 39, 1 (2011).

[12] C. Felser, V. Alijani, J. Winterlik, S. Chadov, and A. K. Nayak, IEEE Trans. Magn. 49, 682 (2013).

[13] C. Felser, L. Wollmann, S. Chadov, G. Fecher, and S. S. P. Parkin, APL Mater. 3, 041518 (2015).

[14] S. Mizukami, A. Sakuma, A. Sugihara, K. Z. Suzuki, and R. Ranjbar, Scr. Mater. 118, 70 (2016).

[15] S. V. Faleev, Y. Ferrante, J. Jeong, M. G. Samant, B. Jones, and S. S. P. Parkin, Phys. Rev. Mater. 1, 024402 (2017).

[16] B. Balke, G. H. Fecher, J. Winterlik, and C. Felser, Appl. Phys. Lett. 90, 152504 (2007).

[17] J. Winterlik, B. Balke, G. Fecher, C. Felser, M. Alves, F. Bernardi, and J. Morais, Phys. Rev. B 77, 054406 (2008).

[18] H. Kurt, K. Rode, M. Venkatesan, P. S. Stamenov, and J. M. D. Coey, Phys. Rev. B 83, 020405(R) (2011).

[19] H. Kurt, K. Rode, M. Venkatesan, P. Stamenov, and J. M. D. Coey, Phys. Status Solidi (B) 248, 2338 (2011).

[20] Q. Ma, A. Sugihara, K. Suzuki, X. Zhang, T. Miyazaki, and S. Mizukami, Spin 4, 1440024 (2014).

[21] J. Jeong, Y. Ferrante, S. V. Faleev, M. G. Samant, C. Felser, and S. S. P. Parkin, Nat. Commun. 7, 10276 (2016).

[22] A. Sugihara, S. Mizukami, Y. Yamada, K. Koike, and T. Miyazaki, Appl. Phys. Lett. 104, 132404 (2014).

[23] S. Mizukami, A. Sakuma, A. Sugihara, T. Kubota, Y. Kondo, H. Tsuchiura, and T. Miyazaki, Appl. Phys. Express 6, 123002 (2013).
[24] A. Sugihara, K. Suzuki, S. Mizukami, and T. Miyazaki, J. Phys. D: Appl. Phys. 48, 164009 (2015).

[25] H. Kurt, N. Baadji, K. Rode, M. Venkatesan, P. Stamenov, S. Sanvito, and J. M. D. Coey, Appl. Phys. Lett. 101, 132410 (2012).

[26] T. Gasi, A. K. Nayak, J. Winterlik, V. Ksenofontov, P. Adler, M. Nicklas, and C. Felser, Appl. Phys. Lett. 102, 202402 (2013).

[27] T. Gasi, A. K. Nayak, M. Nicklas, and C. Felser, J. Phys. D: Appl. Phys. 113, 17E301 (2013).

[28] X. D. Tang, W. H. Wang, W. Zhu, E. K. Liu, G. H. Wu, F. B. Meng, H. Y. Liu, and H. Z. Luo, Appl. Phys. Lett. 97, 242513 (2010).

[29] A. K. Nayak, M. Nicklas, S. Chadov, P. Khuntia, C. Shekhar, A. Kalache, M. Baenitz, Y. Skourski, V. K. Guduru, A. Puri, U. Zeitler, J. M. D. Coey, and C. Felser, Nat. Mater. 14, 679 (2015).

[30] S. Ener, J. Kroder, K. P. Skokov, and O. Gutfleisch, J. Alloys Compd. 683, 198 (2016).

[31] S. Ouardi, T. Kubota, G. H. Fecher, R. Stinshoff, S. Mizukami, T. Miyazaki, E. Ikenaga, and C. Felser, Appl. Phys. Lett. 101, 242406 (2012).

[32] C. Fowley, S. Ouardi, T. Kubota, O. Yildirim, A. Neudert, K. Lenz, V. Sluka, J. Lindner, J. M. Law, S. Mizukami, G. H. Fecher, C. Felser, and A. M. Deac, J. Phys. D: Appl. Phys. 48, 164006 (2015).

[33] R. Sahoo, L. Wollmann, S. Selle, T. Höche, B. Ernst, A. Kalache, C. Shekhar, N. Kumar, S. Chadov, C. Felser, S. S. P. Parkin, and A. K. Nayak, Adv. Mater. 28, 8499 (2016).

[34] H. Kurt, K. Rode, P. Stamenov, M. Venkatesan, Y.-C. Lau, E. Fonda, and J. M. D. Coey, Phys. Rev. Lett. 112, 027201 (2014).

[35] N. Thiyagarajah, Y.-C. Lau, D. Betto, K. Borisov, J. M. D. Coey, P. Stamenov, and K. Rode, Appl. Phys. Lett. 106, 122402 (2015).

[36] O. Meshcheriakova, A. Köhler, S. Ouardi, Y. Kondo, T. Kubota, S. Chandra, J. Karel, C. V. Barbosa, R. Stinshoff, R. Sahoo, S. Ueda, E. Ikenaga, S. Mizukami, S. Chadov, D. Ebke, G. H. Fecher, and C. Felser, J. Phys. D: Appl. Phys. 48, 164008 (2015).

[37] O. Meshcheriakova, S. Chadov, A. K. Nayak, U. K. Rößler, J. Kübler, G. André, A. A. Tsirlin, J. Kiss, S. Hausdorf, A. Kalache, W. Schnelle, M. Nicklas, and C. Felser, Phys. Rev. Lett. 113, 087203 (2014).

[38] S. Chadov, S. W. D’Souza, L. Wollmann, J. Kiss, G. H. Fecher, and C. Felser, Phys. Rev. B 91, 094203 (2015).

[39] A. Niesen, C. Sterwerf, M. Glas, J.-M. Schmalhorst, and G. Reiss, IEEE Trans. Magn. 52, 14 (2016).

[40] D. Betto, Y.-C. Lau, K. Borisov, K. Kummer, N. B. Brookes, P. Stamenov, J. M. D. Coey, and K. Rode, Phys. Rev. B 96, 024408 (2017).

[41] A. Kalache, A. Markou, S. Selle, T. Höche, R. Sahoo, G. H. Fecher, and C. Felser, APL Mater. 5, 096102 (2017). 
[42] B. S. Yang, L. N. Jiang, W. Z. Chen, P. Tang, J. Zhang, X.-G. Zhang, Y. Yan, and X. F. Han, Appl. Phys. Lett. 112, 142403 (2018).

[43] A. Koeba, T. Shima, and M. Doi, Jpn. J. Appl. Phys. 55, 07 MC04 (2016).

[44] V. Alijani, S. Ouardi, G. H. Fecher, J. Winterlik, S. S. Naghavi, X. Kozina, G. Stryganyuk, C. Felser, E. Ikenaga, Y. Yamashita, S. Ueda, and K. Kobayashi, Phys. Rev. B 84, 224416 (2011).

[45] F. de Groot and A. Kotani, Core Level Spectroscopy of Solids (CRC Press, Bota Raton, 2008).
[46] A. Kundu and S. Ghosh, Intermetallics 93, 209 (2018).

[47] J. Karel, F. Casoli, L. Nasi, P. Lupo, R. Sahoo, B. Ernst, A. Markou, A. Kalache, R. Cabassi, F. Albertini, and C. Felser, Appl. Phys. Lett. 111, 182405 (2017).

[48] A. Köhler, I. Knez, D. Ebke, C. Felser, and S. S. P. Parkin, Appl. Phys. Lett. 103, 162406 (2013).

[49] K. Z. Suzuki, R. Ranjbar, A. Sugihara, T. Miyazaki, and S. Mizukami, Jpn. J. Appl. Phys. 55, 010305 (2016).

[50] S. Khmelevskyi, A. B. Shick, and P. Mohn, Appl. Phys. Lett. 109, 222402 (2016).

[51] S. K. Saha, Z. Liu, and G. Dutta, Sci. Rep. 7, 3 (2017). 